\title{
Modelado del Régimen Dinámico y Análisis de Sensibilidad de una Máquina Trifásica de Inducción Magnética
}

\author{
Leopoldo Simón y José M. Monzón \\ Departamento de Ingeniería Eléctrica, Universidad de Las Palmas de Gran Canaria, Islas Canarias- \\ España (e-mail: Isimon@pas.ulpgc.es)
}

Recibido Oct. 29, 2009; Aceptado Ene. 19, 2010; Versión Final recibida Feb. 12, 2010

\section{Resumen}

En este artículo se presenta un análisis teórico de una máquina de inducción magnética trifásica, que incluye una identificación paramétrica a partir de su circuito equivalente. En el planteamiento se ha hecho un modelado singular de la parte mecánica en el mismo circuito equivalente, así como de la máquina en su conjunto. Las ecuaciones obtenidas se han planteado en variables de estado, haciendo posible que las ecuaciones diferenciales puedan ser expresadas de forma que sus primeras derivadas se encuentren despejadas, llegándose a un método unificado para su análisis y simulación numérica. Mediante unos programas desarrollados en $\mathrm{C}++$, se ha hecho un completo análisis de sensibilidad para el modelo permanente y el modelo dinámico. Esto ha permitido observar y estudiar la influencia e interrelación de sus parámetros.

Palabras clave: identificación paramétrica, circuito equivalente, variables de estado, análisis de sensibilidad

\section{Dynamic Modeling and Sensitivity Analysis of a Magnetic Three-Phase Induction Machine}

\begin{abstract}
In this paper a theoretical analysis of magnetic three-phase induction machine is presented, including an identification parameter from its equivalent circuit. The approach has become an outstanding model for the mechanical part in the same equivalent circuit, and the machine as a whole. The equations obtained have been raised as state variables. Then, the differential equations can be expressed in function of the first derivative, obtaining a unified method for the simulation and numerical analysis. Through programs developed in $\mathrm{C}++$, a complete sensitivity analysis for the steady state and dynamic models was developed. This allowed to observe and to study the influence and relationship between these parameters.
\end{abstract}

Keywords: identification parameter, equivalent circuit, state variables, sensitivity analysis 


\section{INTRODUCCIÓN}

Las máquinas de inducción presentan un difícil problema de control. Esto es debido principalmente a que el modelo dinámico del sistema no es lineal, a que las intensidades en el rotor no son medibles, y al calentamiento resistivo, que hace variar el valor de las resistencias rotóricas considerablemente con su correspondiente efecto en la dinámica del sistema. A menudo es necesario hacer in-situ, una auditoría o evaluación de motores existentes en una industria, con el fin de conocer su rendimiento y minimizar costes; más aún, existe una situación de diseño previo a la construcción de un motor de inducción, donde es necesario el conocimiento de sus parámetros, para evaluarlos y ajustarlos a las especificaciones de construcción que se demandan (Molinar et al., 1996). También, en lo que respecta al control, se hace necesario tener un modelo paramétrico en bucle que sea observable, y que ayude en los procesos de diseño a la optimización de los dispositivos controladores. Por lo tanto, conocer los parámetros que permitan la identificación de las máquinas, y como estos influyen entre sí, es una tarea que se plantea hoy como una necesidad para su correcto cálculo, control y evaluación.

Clásicamente, para las máquinas utilizadas en los sistemas de control, se aplica la respuesta transitoria incremental a la hora del análisis de su estabilidad (Singh et al., 2005). Pero en la respuesta transitoria incremental, las ecuaciones son linealizadas entorno al estado de operación nominal, en el cual las derivadas son nulas, sin embargo, una de las aportaciones que se incluyen en este artículo consiste en la utilización del método de variable de estado, para este tipo de análisis.

La razón de plantear el circuito equivalente en variables de estado, es que sus ecuaciones diferenciales se pueden expresar en un formato especial, donde las derivadas primeras de las variables de estado están despejadas, ofreciendo un método unificado para el estudio de sistemas para su análisis y simulación. Entre sus características fundamentales está el hecho de que se trabaja directamente en el dominio del tiempo, sin tener que pasar a dominios transformados y además, la ecuación en diferencias de orden n que determina al sistema, se descompone en $n$ ecuaciones de orden uno que admiten un tratamiento común, independientemente del sistema que esté siendo considerado.

La aportación de este artículo consiste en la deducción de un modelo de circuito equivalente de la máquina de inducción (Fig. 1) (Meeker, 2002; Meisel, 1984). Del mismo se han planteado las ecuaciones de equilibrio tanto de la parte eléctrica como de la mecánica, la cual ha sido también modelada mediante un circuito equivalente. Estas ecuaciones de equilibrio se han resuelto y se han dispuesto de forma que queden planteadas en variables de estado (Marungsri et al., 2006). Para su resolución se ha utilizado el método de Runge-Kutta-Fehlberg (RKF) (Nicolet y Delincé, 1996; Shampine, 2005) para resolver ecuaciones diferenciales no lineales.

El programa utilizado es un algoritmo de propósito general escrito en lenguaje $\mathrm{C}++$, de código libre y abierto, siendo adaptado para este fin. La compilación se ha hecho mediante el compilador de licencia pública $\mathrm{Gcc}++$ que es un compilador de lenguaje $\mathrm{C} / \mathrm{C}++$. Para las simulaciones además del algoritmo RKF, se ha desarrollado íntegramente un programa para los análisis del modelo en régimen permanente, realizado en el software de cálculo científico orientado a la computación numérica Scilab (Scilab Group, 1997). Toda la representación gráfica de los datos obtenidos se ha hecho mediante el programa Gnuplot, también de licencia pública. El artículo en su conjunto ha sido elaborado en el procesador de texto para documentos científicos Latex, bajo el sistema operativo openSUSE-Linux.

\section{MODELO PARAMÉTRICO DEL MOTOR DE INDUCCIÓN EN RÉGIMEN DINÁMICO}

La identificación paramétrica de la máquina de inducción, se hace partiendo de las ecuaciones (1) y (2) (Meisel, 1984; Mirafzal et al., 2009; Steven y Steven, 1999). Se ha utilizado la transformación de Park para la conversión de las tres fases del motor de inducción en componentes más sencillas d-q0 , ya que utilizan magnitudes reales contrariamente a la transformación de $\mathrm{Ku}$, que trabaja en el dominio complejo (Laczynski y Mertens, 2009; Marungsri et al., 2006). 


$$
\begin{aligned}
& {\left[\begin{array}{c}
v_{d s} \\
v_{q s} \\
v_{d r} \\
v_{q r}
\end{array}\right]=\left[\begin{array}{cccc}
R_{s}+L_{s} p & 0 & M_{s r} p & 0 \\
0 & R_{s}+L_{s} p & 0 & M_{s r} p \\
M_{s r} p & n M_{s r} \omega_{r} & R_{r}+L_{r} p & n L_{r} \omega_{r} \\
-n M_{s r} \omega_{r} & M_{s r} p & -n L_{r} \omega_{r} & R_{r}+L_{r} p
\end{array}\right]\left[\begin{array}{c}
i_{d s} \\
i_{q s} \\
i_{d r} \\
i_{q r}
\end{array}\right]} \\
& T_{r}=(J p+D) \omega_{r}+n M_{s r}\left(i_{d s} i_{q r}-i_{q s} i_{d r}\right)
\end{aligned}
$$

Del modelo planteado de la máquina de inducción, se obtiene el circuito equivalente representado en la Fig. 1, del cual se deducirán todas las variables de estado.

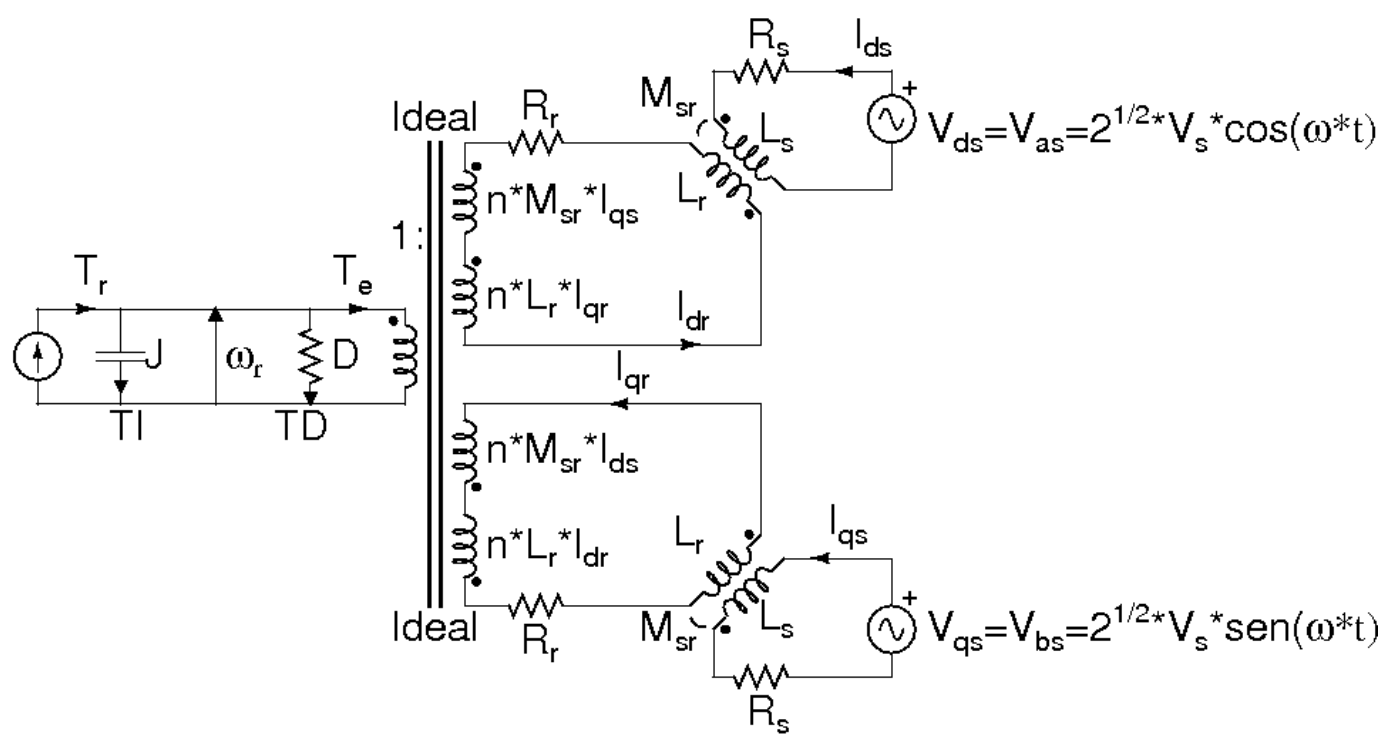

Fig. 1: Circuito equivalente de la máquina de inducción.

Estas variables son, al ser un circuito tiempo invariante, las intensidades de las bobinas y las tensiones de los condensadores, o sea $x(t)=\left(I_{d s}(t), I_{d r}(t), I_{q r}(t), I_{q s}(t), \omega_{r}\right)$, respectivamente. Si el circuito fuera tiempo variante, las variables de estado serían los flujos en las bobinas y las cargas en los condensadores. De igual forma, el vector $u(t)=\left(v_{d s}, v_{q s}, T_{r}\right)$, se corresponde con las fuentes independientes de tensión e intensidad del circuito de la Fig. 1.

Las excitaciones del circuito equivalente están formadas por las fuentes de tensión en la parte eléctrica y la fuente de intensidad en la parte mecánica. Ambas partes están separadas por un transformador ideal.

La parte mecánica se ha modelado de forma singular mediante un circuito equivalente. Como elementos en paralelo se han modelado el momento de inercia $\mathrm{J}$ como un condensador y el coeficiente angular de fricción viscosa D, como una conductancia. El par resistente aplicado se modela mediante una excitación formada por una fuente de intensidad de valor $T_{r}$, la velocidad angular del rotor como una tensión aplicada $\omega_{\mathrm{r}} \mathrm{y}$ el par de origen eléctrico $\mathrm{T}_{\mathrm{e}}$.

El modelado de la parte eléctrica está constituido por la resistencias de los devanados del estátor $R_{s}$ y del rotor $R_{r}$, la autoinducción de los mismos como $L_{s}$ y $L_{r}$, la inducción mutua entre estátor y rotor $M_{s r}$, las intensidades tanto del eje directo $I_{d s}$, $I_{d r}$ como en cuadratura $I_{q s}$, $I_{q r}$, y en el estátor, las fuentes de tensión como excitaciones en ambos ejes $\mathrm{V}_{\mathrm{ds}} \mathrm{y}_{\mathrm{qs}}$, siendo $\mathrm{n}$ el número de pares de polos.

Del modelo de la máquina de inducción y de su circuito equivalente, y de las ecuaciones correspondientes para los devanados directos y en cuadratura del estátor y del rotor, se han planteado las ecuaciones de equilibrio para la parte eléctrica (3) y para la parte mecánica (4). 
Se han obtenido las derivadas primeras en las ecuaciones diferenciales no lineales y agrupados los términos, se plantean las variables de estado en forma matricial quedando según las expresiones (3) y (4). Al ser un circuito tiempo invariante, estas se corresponden con las intensidades en las bobinas y las tensiones en los condensadores.

En las ecuaciones de equilibrio deducidas, aparece también como variable el ángulo de rotación mecánico $\theta$, que aunque no es variable de estado, su función estará presente en la transformación inversa o equivalente con el modelo trifásico.

$$
\begin{aligned}
& \frac{d}{d t}\left[\begin{array}{c}
i_{d s} \\
i_{q s} \\
i_{d r} \\
i_{q r}
\end{array}\right]=-\frac{1}{L_{s} L_{r}-\left(M_{s r}\right)^{2}}\left[\begin{array}{cccc}
n L_{r} R_{s} & -n\left(M_{s r}\right)^{2} \omega_{r} & -M_{s r} R_{r} & -M_{s r} L_{r} \omega_{r} \\
n\left(M_{s r}\right)^{2} \omega_{r} & L_{r} R_{s} & n M_{s r} L_{r} \omega_{r} & -M_{s r} R_{r} \\
-M_{s r} R_{s} & n L_{s} M_{s r} \omega_{r} & L_{s} R_{r} & n L_{s} L_{r} \omega_{r} \\
-n L_{s} M_{s r} \omega_{r} & -M_{s r} R_{s} & -n L_{s} L_{r} \omega_{r} & L_{s} R_{r}
\end{array}\right]\left[\begin{array}{l}
i_{d s} \\
i_{q s} \\
i_{d r} \\
i_{q r}
\end{array}\right]+ \\
& +\frac{1}{L_{s} L_{r}-\left(M_{s r}\right)^{2}}\left[\begin{array}{cc}
L_{r} & 0 \\
0 & L_{r} \\
-M_{s r} & 0 \\
0 & -M_{s r}
\end{array}\right]\left[\begin{array}{c}
v_{d s} \\
v_{q s}
\end{array}\right] \\
& \frac{d}{d t}\left[\begin{array}{c}
\omega_{r} \\
\theta
\end{array}\right]=\left[\begin{array}{c}
-\frac{1}{J} D \omega_{r}-\frac{1}{J} n M_{s r}\left(i_{d s} i_{q r}-i_{q s} i_{d r}\right) \\
\omega_{r}
\end{array}\right]+\left[\begin{array}{c}
\frac{1}{J} \\
0
\end{array}\right] T_{r}
\end{aligned}
$$

El sistema general (3) y (4) responde a un sistema de ecuaciones diferenciales algebraicas (EDA) (Nicolet y Delincé, 1996) y viene referenciado por

$$
A \dot{y}(t)+B y(t)=b(t)
$$

donde $A$ y $B$ son matrices de coeficientes, $b$ es el vector del segundo miembro, $y(t)$ es el vector desconocido, $t$ es el tiempo y el punto es la derivada respecto del tiempo. A y B pueden depender de $\mathrm{y}(\mathrm{t})$ en el caso de sistemas no lineales, por ejemplo B depende de las incógnitas en el sistema de ecuaciones (7). La solución numérica del sistema (5) requiere una discretización del tiempo. Un esquema simple viene dado por (6)

$$
A \frac{y_{n+1}-y_{n}}{\Delta t}+B\left(\alpha y_{n+1}+(1-\alpha) y_{n}\right)=\alpha b_{n+1}+(1-\alpha) b_{n}
$$

Los índices $n$ y $n+1$ se refieren a cantidades en el tiempo $t_{n}$ y $t_{n+1}=t_{n}+\Delta t$ respectivamente. Las diferentes opciones del parámetro $\alpha$ se obtienen por métodos clásicos $(\alpha=1$, es implícito (hacia atrás) de Euler, $\alpha=0$ es explícito (hacia adelante) de Euler, $\alpha=1 / 2$ es Crack-Nicolson, $\alpha=2 / 3$ es Galerkin).

En este artículo el sistema general viene dado por una ecuación diferencial ordinaria (EDO) que es un caso especial de las EDA, donde la matriz A es la unidad. Si $k=\frac{1}{L_{s} L_{r}-\left(M_{s r}\right)^{2}}$, las matrices de coeficientes planteadas en el sistema general (5) quedan determinadas de la siguiente forma en (7): 


$$
A=\left[\begin{array}{ccccc}
1 & 0 & 0 & 0 & 0 \\
0 & 1 & 0 & 0 & 0 \\
0 & 0 & 1 & 0 & 0 \\
0 & 0 & 0 & 1 & 0 \\
0 & 0 & 0 & 0 & 1
\end{array}\right] ; \quad B=k\left[\begin{array}{ccccc}
n L_{r} R_{s} & -n\left(M_{s r}\right)^{2} \omega_{r} & -M_{s r} R_{r} & -M_{s r} L_{r} \omega_{r} & 0 \\
n\left(M_{s r}\right)^{2} \omega_{r} & L_{r} R_{s} & n M_{s r} L_{r} \omega_{r} & -M_{s r} R_{r} & 0 \\
-M_{s r} R_{s} & n L_{s} M_{s r} \omega_{r} & L_{s} R_{r} & n L_{s} L_{r} \omega_{r} & 0 \\
-n L_{s} M_{s r} \omega_{r} & -M_{s r} R_{s} & -n L_{s} L_{r} \omega_{r} & L_{s} R_{r} & 0 \\
\frac{1}{k} \frac{1}{J} n M_{s r} i_{q r} & -\frac{1}{k} \frac{1}{J} n M_{s r} i_{d r} & 0 & 0 & \frac{1}{k} \frac{D}{J}
\end{array}\right]
$$

$$
b=k\left[\begin{array}{ccc}
L_{r} & 0 & 0 \\
0 & L_{r} & 0 \\
-M_{s r} & 0 & 0 \\
0 & -M_{s r} & 0 \\
0 & 0 & \frac{1}{k} \frac{1}{J}
\end{array}\right]\left[\begin{array}{c}
v_{d s}(t) \\
v_{q s}(t) \\
T_{r}(t)
\end{array}\right]
$$

\section{RESULTADOS Y DISCUSIÓN}

Como método de resolución se ha utilizado el método de Runge-Kutta-Fehlberg (RKF) de $4^{\circ}$ y $5^{\circ}$ orden, de paso fijo (Nicolet y Delincé, 1996; Shampine, 2005). Este método ha resuelto satisfactoriamente las ecuaciones diferenciales no lineales. El paso utilizado ha sido $\Delta t=0.0001$ segundos, para un rango de tiempo comprendido entre $t_{o}=0$ y $t_{f}=2$ segundos. Las condiciones iniciales de las variables de estado son las intensidades $I_{d s}(t)=0, I_{d r}(t)=0, I_{q s}(t)=0, I_{q r}(t)=0$, en amperios y la velocidad inicial del rotor $\omega_{r}=0$ en radianes/segundo.

Se han recopilado una serie de gráficas con las que se pretende mostrar el comportamiento del modelo de la máquina de inducción; en primer lugar, ante la variación de las magnitudes de algunos parámetros constantes, y segundo lugar, en la comparación entre el modelo dinámico y el modelo clásico par-velocidad en régimen permanente.

Tabla 1: Valores correspondientes a cuatro experiencias en el análisis de sensibilidad realizado.

\begin{tabular}{|c|c|c|c|c|}
\hline & $\mathrm{E} 1$ & $\mathrm{E} 2$ & $\mathrm{E} 3$ & $\mathrm{E} 4$ \\
\hline $\mathrm{R}_{\mathrm{r}}(\Omega)$ & $3 / 6 / 9$ & 6 & 6 & 6 \\
\hline $\mathrm{R}_{\mathrm{s}}(\Omega)$ & 2.9 & $1.5 / 2.9 / 4.9$ & 2.9 & 2.9 \\
\hline $\mathrm{K}_{\mathrm{sr}}$ & 0.980 & 0.980 & $0.965 / 0.980 / 0.995$ & 0.980 \\
\hline $\mathrm{J}$ & 1.0 & 1.0 & 1.0 & $0.5 / 1.0 / 1.5$ \\
\hline $\mathrm{L}_{\mathrm{r}}(\mathrm{H})$ & 0.880 & 0.880 & 0.880 & 0.880 \\
\hline $\mathrm{L}_{\mathrm{s}}(\mathrm{H})$ & 0.880 & 0.880 & 0.880 & 0.880 \\
\hline $\mathrm{p}$ & 1 & 1 & 1 & 1 \\
\hline $\mathrm{V}_{\mathrm{s}}(\mathrm{V})$ & 1200 & 1200 & 1200 & 1200 \\
\hline $\mathrm{F}(\mathrm{Hz})$ & 50 & 50 & 50 & 50 \\
\hline
\end{tabular}

En una serie de experiencias, se analiza la respuesta del modelo para variaciones en magnitudes constantes. Como situación inicial, se parte de los parámetros ya definidos de una máquina real. La Tabla 1, recoge los valores iniciales más significativos no nulos, y las cuatro experiencias correspondientes a la variación de los parámetros correspondientes a la resistencia del rotor $R_{r}$, la resistencia del estátor $R_{s}$, el coeficiente de acoplamiento entre el rotor y el estátor $K_{s r}$ y el momento de inercia $\mathrm{J}$, que son magnitudes constantes.

A cada uno de ellos se le hace variar con un valor superior y otro inferior al de definición. Como resultados se obtendrán los valores del par electromagnético $\mathrm{T}$ como magnitud derivada, y las variables de estado definidas por la velocidad de rotor $\omega_{\mathrm{r}}$, la intensidad del estátor $\mathrm{I}_{\mathrm{s}}$, y la intensidad del rotor $\mathrm{I}_{\mathrm{r}}$. 
Como ejemplo inicial, las Fig. 2 y 3 representan el transitorio del par electromagnético del modelo dinámico para variaciones de la resistencia rotórica.

En la Fig. 2, se muestra los detalles del transitorio en los primeros instantes de arranque del motor, donde el par oscila llegando a cambiar de signo en contraposición con el régimen permanente.

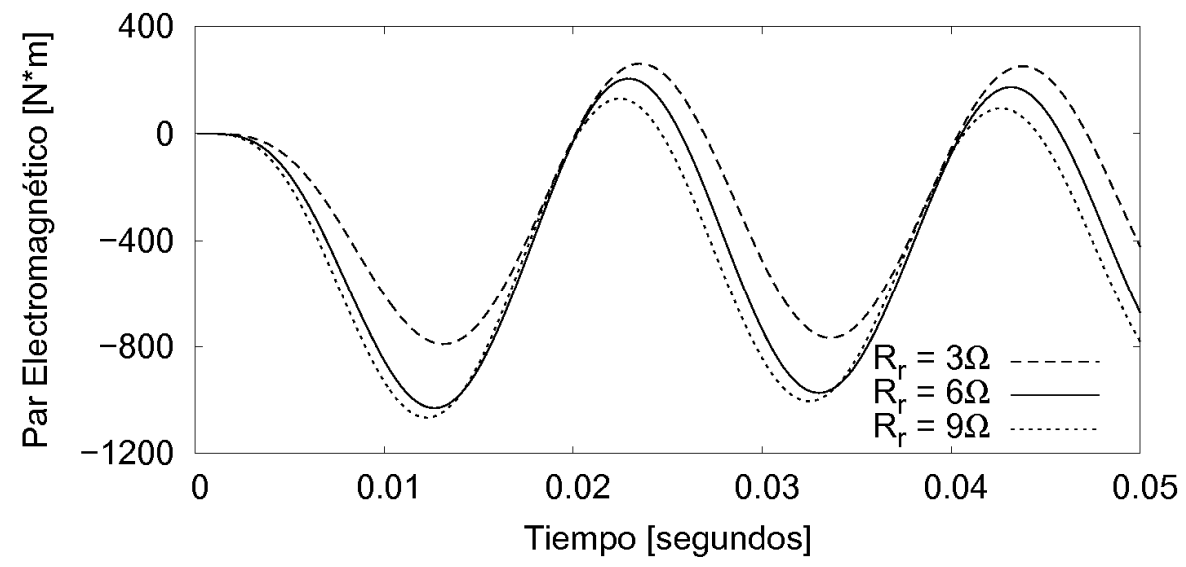

Fig. 2: Primeros instantes de los transitorios del par para distintas resistencias de rotor, según E1.

En la Fig. 3, se puede observar como análogamente al régimen permanente, el valor del par máximo no depende de la resistencia rotórica, influyendo sólo en el tiempo en que se alcanza éste; para valores pequeños de $R_{r}$ se alcanza antes el par máximo. Con lo cual, el par máximo que puede generar una máquina de inducción para una excitación dada, es independiente de la resistencia del rotor $R_{r}$, y el deslizamiento para el que tiene lugar el par máximo, puede ajustarse con dicha resistencia.

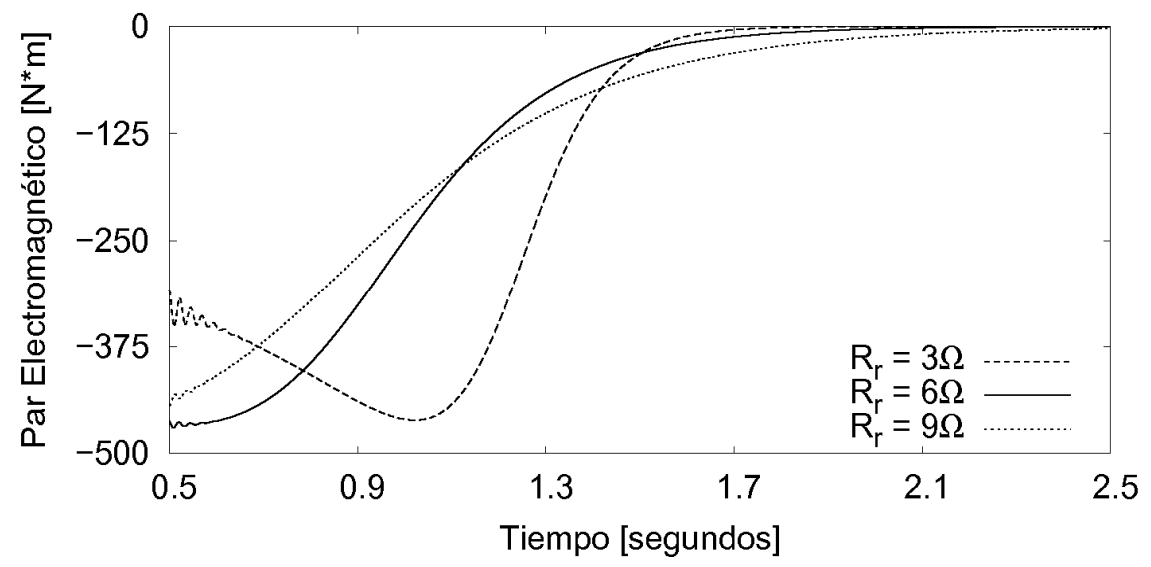

Fig. 3: Valores máximos del par desplazados en el tiempo según los valores de resistencia de rotor.

En la Fig. 4 se observa perfectamente la diferencia entre el sobreamortiguamiento del modelo clásico par-velocidad del régimen permanente donde se ha considerado que los transitorios eléctricos son mucho más rápidos que los mecánicos, frente al comportamiento real en el régimen dinámico del par electromagnético. Se observa las oscilaciones que se producen en el par en los instantes iniciales. 


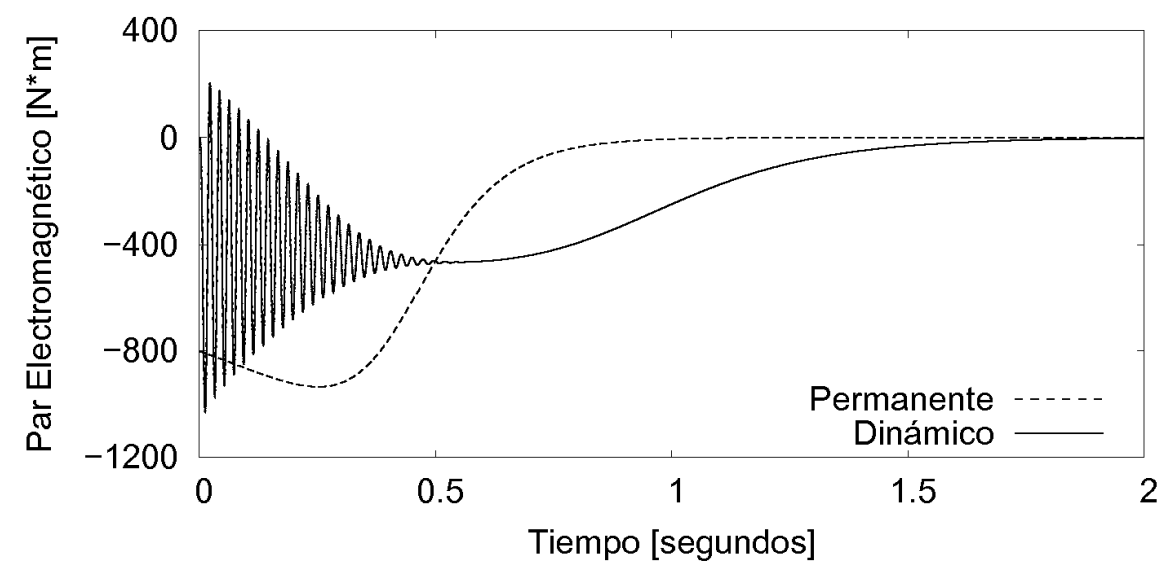

Fig. 4: Comparación entre el modelo dinámico y el modelo permanente del par electromagnético.

En la Fig. 5 se observa como en el modelo dinámico se alcanza la velocidad máxima más lentamente que en el régimen permanente, como consecuencia del comportamiento más realista del régimen dinámico.

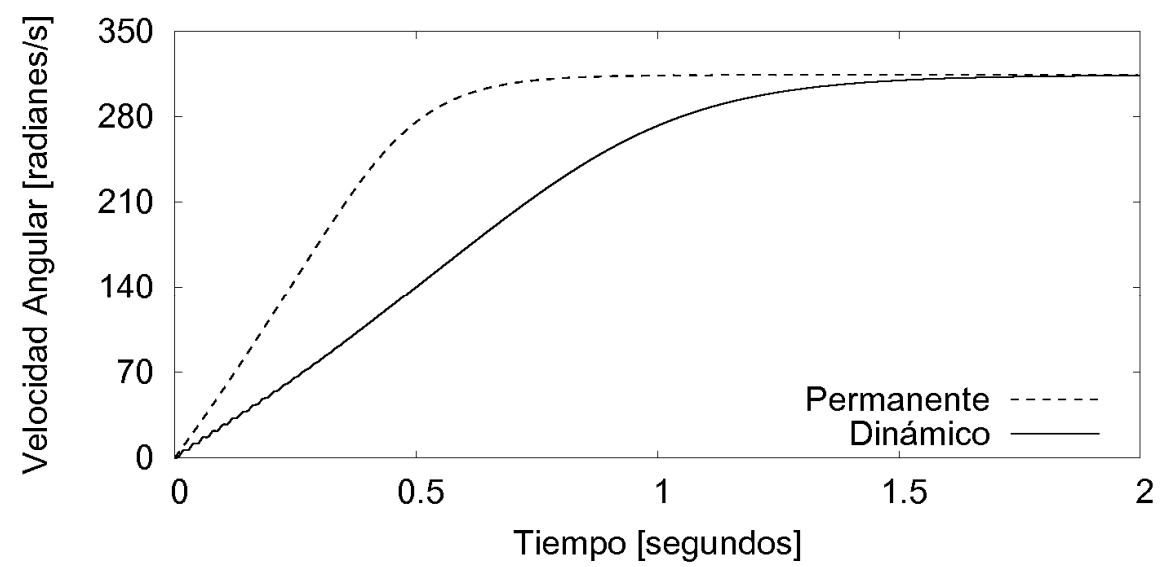

Fig. 5: Comparación entre el modelo dinámico y el modelo permanente de la velocidad.

La Fig. 6, corresponde al transitorio de la intensidad de la fase 'a' del rotor sometido a tres escalones en bucle abierto.

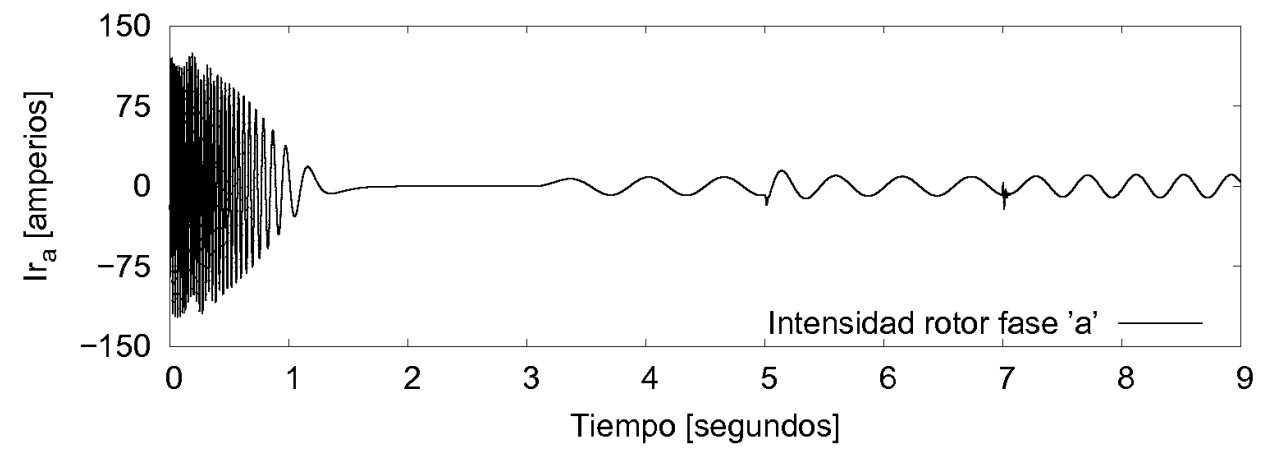

Fig. 6: Transitorio de la intensidad de la fase 'a' del rotor, con tres escalones.

Estos escalones producen: a los tres segundos un escalón donde varía el par resistente de 0 a -45 $\mathrm{N}^{*} \mathrm{~m}$; a los cinco segundos un escalón donde varía la frecuencia de 50 a $52 \mathrm{~Hz}$; y por último un escalón a los siete segundos donde se produce una variación en la tensión del estátor de 1200 a 1000 voltios. De esta forma el sistema se ha sometido a cambios en su dinámica que se han podido simular perfectamente con el planteamiento circuital y numérico propuesto. 


\section{CONCLUSIONES}

Se ha deducido un circuito equivalente de la máquina de inducción y un circuito equivalente singular de la parte mecánica. Del circuito equivalente se ha hecho un planteamiento de las ecuaciones de equilibrio en variables de estado. De entre los muchos métodos existentes de resolución, se ha utilizado el método de RKF de paso fijo, lográndose una herramienta para el análisis del modelo de la máquina de inducción. Se somete a estudio la variación de parámetros concentrados a partir del circuito equivalente, y se compara el modelo dinámico con el modelo clásico par-velocidad, observándose la importante diferencia que existe entre ambos en el transitorio, siendo la respuesta de ambos coincidente en el régimen permanente. Cuando se tiene una $R_{r}$ elevada, se obtiene un buen par de arranque (deslizamiento=1). El análisis de sensibilidad demuestra que el par máximo, no depende de la $R_{r}$. También se han obtenido respuestas de magnitudes derivadas como el par electromagnético así como la velocidad angular para ambos regímenes. Se muestra un desarrollo de la dinámica del sistema sometido a una serie de perturbaciones en bucle abierto, lo que permitirá posteriores aplicaciones en el análisis de sistemas de control.

\section{REFERENCIAS}

Laczynski T. y A. Mertens, Predictive Stator Current Control for Medium Voltage Drives With LC Filters, IEEE Trans. on Power Electronics, 24(11), Noviembre (2009).

Marungsri, B., N. Meeboon y A. Oonsivilai, Dynamic Model Identification of Induction Motors using Intelligent Search Techniques with taking Core Loss into Account, $6^{\text {th }}$ WSEAS International Conference on Power Systems, 108-115, Lisboa, Portugal 22 al 24 de Septiembre (2006).

Meeker, D., Induction Motor Example, IEEE dmeeker@ieee.org (2002).

Meisel, J., Principles of Electromechanical Energy Conversion, Robert E. Krieger Publishing Company, Florida USA (1984).

Mirafzal, B., G. L. Skibinski y R. M. Tallam, Determination of Parameters in the Universal Induction Motor Model, IEEE Trans. Industry Applications, 45, 1, 142-151, Enero-Febrero (2009).

Molinar, D., y otros cuatro autores, Calculation of Two-Axis Induction Motor Model Parameters using Finite Elements, IEEE Trans. Magnetics, 12(2), 133-142 (1997).

Nicolet, A. y F. Delincé, Implicit Runge-Kutta Methods for Transient Magnetic Field Computation, IEEE Trans. Magnetics, 32(3), 1405-1408 (1996).

Scilab Group, Introduction to scilab: User's guide, Tech. Rep., Unité de recherché de Rocquencourt Project Méta-2, http://www-rocq.inria.fr , http://www.scilab.org (1997).

Shampine, L.F., Error Estimation and Control for ODEs, Journal of Scientific Computing, ISSN: 08857474 (en línea), 25(1), 3-16, Mathematics Department Southern Methodist University, Dallas, USA (2005).

Singh, B., G. Bhuvaneswari y V. Garg, T-Connected Autotransformer-Based 24-Puls AC-DC Converter for Variable Frequency Induction Motor Drives, IEEE Trans. on Energy Conversion, 21(3), Septiembre (2006).

Steven, R. y B. Steven, Identification of Induction Motor Parameters from Transient Stator Current Measurements. Member, IEEE Trans. Industrial Electronics, 0278-0046, 46(1), 139-149 (1999). 